OPEN ACCESS

Edited by:

Lijun Wang,

Institute of Botany-The Chinese

Academy of Sciences, China

Reviewed by:

Xiangfeng Wang,

The University of Arizona, USA

Weirong $X u$,

Ningxia University, China

*Correspondence:

Yan Xu

yan.xu@nwsuaf.edu.cn

Specialty section:

This article was submitted to Plant Biotic Interactions,

a section of the journal Frontiers in Plant Science

Received: 16 October 2015 Accepted: 22 April 2016 Published: 31 May 2016

Citation:

Han L, Weng K, Ma H, Xiang G, Li Z,

Wang Y, Liu G and Xu Y (2016) Identification and Characterization of

Erysiphe necator-Responsive

MicroRNAs in Chinese Wild Vitis pesudoreticulata by High-Throughput Sequencing. Front. Plant Sci. 7:621. doi: 10.3389/fpls.2016.00621

\section{Identification and Characterization of Erysiphe necator-Responsive MicroRNAs in Chinese Wild Vitis pseudoreticulata by High-Throughput Sequencing}

\author{
Lijuan Han 1,2,3, Kai Weng 1,2,3, Hui Ma 1,2,3, Gaoqing Xiang 1, 2, 3, Zhiqian Li 1, 2, 3, \\ Yuejin Wang ${ }^{1,2,3}$, Guotian Liu ${ }^{1,2,3}$ and Yan $X u^{1,2,3 *}$
}

1 State Key Laboratory of Crop Stress Biology for Arid Areas, College of Horticulture, Northwest A\&F University, Yangling, China, ${ }^{2}$ College of Horticulture, Northwest A\&F University, Yangling, China, ${ }^{3}$ Key Laboratory of Horticultural Plant Biology and Germplasm Innovation in Northwest China, Ministry of Agriculture, College of Horticulture, Northwest A\& F University, Yangling, China

Grapevine powdery mildew is one of the most damaging fungal diseases. Therefore, a precise understanding of the grapevine disease resistance system becomes a subject of significant importance. Plant microRNAs(miRNAs) have been implicated to play regulatory roles in plant biotic stress responses. In this study, high-throughput sequencing and miRDeep-P were employed to identify miRNAs in Chinese wild Vitis pseudoreticulata leaves following inoculation with Erysiphe necator. Altogether, 126 previously identified microRNAs and 124 novel candidates of miRNA genes were detected. Among them, 43 conserved miRNAs belong to 20 families and 23 non-conserved but previously-known miRNAs belong to 15 families. Following $E$. necator inoculation, 119 miRNAs were down-regulated and 131 were up-regulated. Furthermore, the expression changes occurring in 32 miRNAs were significant. The expression patterns of some miRNAs were validated by semi-quantitative RT-PCR and qRT-PCR. A total of 485 target genes were predicted and categorized by Gene Ontology (GO). In addition, 14 vvi-miRNAs were screened with 36 targets which may be involved in powdery mildew resistance in grape. Highly accumulated vi-NewmiR2118 was detected from accession "Baihe-35-1," whose targets were mostly NBS-LRR resistance genes. It was down-regulated rapidly and strongly in "Baihe-35-1" leaves after inoculated with $E$. necator, indicating its involvement in grape powdery mildew resistance. Finally, the study verified interaction between vvi-NewmiR2118 and RPP13 by histochemical staining and GUS fluorescence quantitative assay.

Keywords: Chinese wild Vitis pseudoreticulata, high-throughput sequencing, miRNA identification, powdery mildew resistance, target genes 


\section{INTRODUCTION}

Grapevine is one of the world's oldest and most important fruit crops-culturally, nutritionally and economically. However, many important grapevine varieties are susceptible to pathogen infection, such as Plasmopara viticola (Richter et al., 2006) and Erysiphe necator (Akkurt et al., 2007). The latter is especially detrimental as it occurs in almost all countries and regions where grapes are grown and causes major loss of grape production each year.

Plant miRNAs are a class of $\sim 22$-neucleotide(nt) small noncoding RNAs (Reinhart et al., 2002). Since their discovery in Arabidopsis (Reinhart et al., 2002), a number of miRNAs have been recorded. Though most miRNAs are involved in plant growth and development (Chen, 2005), recent studies have found that miRNAs also play pivotal roles in plant immunity (Mallory and Vaucheret, 2006; Ruiz-Ferrer and Voinnet, 2009; Sunkar et al., 2012). In plants, the first miRNA reported to show disease resistance activity is ath-miRNA393. When induced by flg22, ath-miR393 down regulated the F-box auxin receptors TIR1, AFB2 and AFB3, which further repressed auxin signaling. As a result, the growth of $P$. syringae was restricted on host plants, implying that miRNA-mediated suppression of auxin signaling enables enhanced disease resistance in plants (Navarro et al., 2006). microRNA398 was down-regulated in response to oxidative stress caused by abiotic or biotic stresses (Sunkar et al., 2006; Jagadeeswaran et al., 2009a). After infiltrated with avirulent strain avrRpm1 or avrRpt2, the expression level of ath-miR398 decreased significantly, but one of its targets $\mathrm{Cu} / \mathrm{Zn}$ superoxide dismutase cytosolic CSD1 was elevated, indicating that miR398 involves in the biotic stress regulatory networks (Jagadeeswaran et al., 2009a). Another research found that miR160, miR67 and miR393 were up-regulated obviously while miR825 was down-regulated greatly after inoculating Arabidopsis with Pst DC3000 hrcC for 1 and $3 \mathrm{~h}$ (Fahlgren et al., 2007). In both single mutants dcll and hen1 whose miRNA synthesis was hindered, spores of Pst DC3000 hrcC, Pseudomonas syringae pv. Phaseolicola, P. fluorescens and Escherichia coli proliferated faster than on wild type (Navarro et al., 2008). In Oryza sativa, novel osa-miR7695 targeted an alternatively spliced transcript OsNramp6, a gene contributing to pathogen resistance (Baldrich et al., 2013; Campo et al., 2013). The researchers of this study believed that alternative splicing might be a mechanism adopted by plants of attenuating miRNA-mediated gene regulation. This speculation is proposed by other researches (Yan et al., 2012; Yang et al., 2012) as well, complicating plant immunity mechanisms based on the miRNA regulation. Not only do natural miRNAs act in plant biotic stress regulatory networks, but also some appropriately modified artificial miRNAs can confer resistance to pathogen susceptible plants, which is elegantly proved by Niu et al. (2006). When researchers modified the athmiRNA159 precursor into amiR-P69159 and amiR-HC-Pro159 which respectively targets virus proteins P69 and HC-Pro, transgenic $A$. thaliana plants obtained specific resistance to TYMV and TuMV. Moreover, transgenic plants expressing both artificial miRNAs from a modified dimeric miR159 precursor conferred resistance to both viruses. These results implicate the possibility to use amiRNA-mediated approaches to engineer resistance to multiple viruses in crop plants (Niu et al., 2006).

China is one of the principal centers of origin of Vitis species. Chinese Vitis species, especially wild grapevines have extremely high resistance to many fungi and bacteria diseases. In addition, Chinese Vitis species can be easily crossed with $V$. vinifera, conferring the disease resistance from Chinese Vitis species into other $V$. vinifera like European grapevines (Wang et al., 1998; He, 1999). For example, V. pseudoreticulata accession Baihe-35-1 has been reported several times to be highly resistant to powdery mildew (Wan et al., 2007). Although, some work has been done to analyze the roles of miRNAs in grapevine-pathogen interaction (Alabi et al., 2012), very little is known about miRNA-involved molecular processes that regulate resistance to powdery mildew. In this study, a powdery mildew-resistant accession of Chinese wild Vitis pseudoreticulata Baihe-35-1 was used as materials. This study was aimed to profile the expression of powdery mildew responsive miRNAs in Chinese wild grape. Two sRNA libraries were constructed for inoculated and non-inoculated grapevine leaves. Then the illumina platform was employed for miRNA high-throughput sequencing (Solexa sequencing). Computational analysis was done to screen miRNAs relative to powdery mildew resistance. We also used stem-loop real time qRT-PCR to validate miRNAs differentially expressed. This study identified highly accumulated vvi-NewmiR2118 from $V$. pseudoreticulata. Using susceptible cultivars of Piont Noir as control material, we showed that vvi-NewmiR2118 was down-regulated greatly in "Baihe-35-1" leaves after inoculated with $E$. necator, indicating its involvement in grape powdery mildew resistance. Vvi-NewmiR2118 mainly targeted NBS-LRR resistance genes. Then histochemical staining and GUS fluorescence quantitative assay verified that it interacted RPP13. This study not only enables a better understanding of host-pathogen interactions but also sheds light on new strategies for breeding disease resistance grapevine varieties.

\section{MATERIALS AND METHODS}

\section{Plant Materials and Stress Treatments}

The Chinese wild V. pseudoreticulata accession "Baihe-35-1" used in this study were grown in the Grape Germplasm Resources orchard of Northwest Agriculture and Forest University, Yangling, China. The second to fourth young and healthy leaves from shoot apexes were inoculated with E. necator in field conditions. The inoculation was carried out as described by Weng et al. (2014). Briefly, "Baihe35-1" leaves were pressed against grape leaves already infected with powdery mildew and covered immediately with a paper bag. Sample leaves were collected $0,12,24,48,72,96,120$, and $144 \mathrm{~h}$ after inoculation and stored at $-80^{\circ} \mathrm{C}$. Three-year old clonal Chinese wild $V$. pseudoreticulata accession "Baihe-35-1" and the susceptible control material Pinot Noir were grown in the climate chamber at $16 \mathrm{~h}$ light $/ 8 \mathrm{~h}$ dark at $25-26^{\circ} \mathrm{C}$ for semi-quantitative RT-PCR and real-time qPCR. 


\section{Total RNA Extraction and Small RNA Sequencing}

Total RNA was isolated from each frozen sample using the modified SDS-phenol method (Zhang et al., 2003; Wang et al., 2004). The extracted RNA was then treated with DNAseI to remove genomic DNA. The RNA concentration was detected before and after DNAseI treatment using a NanoDropTM 1000 spectrophotometer (Thermo Fisher Scientific, USA), and its integrity was ascertained visually with $0.6 \%$ agarose gel electrophoresis.

Mix equal total RNAs from each inoculation time as the treated group. Then $1 \mathrm{mg}$ samples of both treated and control RNAs were subjected to small RNA high-throughput sequencing (HTS) with Solexa sequencing technology at the Beijing Genomics Institute (BGI), Shenzhen, China.

\section{Predict miRNAs and Their Targets from V. pseudoreticulata}

miRDeep-P (Yang and Li, 2011) is an effective tool for plant miRNA searching, so it was used in this study to determine and analyze miRNAs in "Baihe-35-1" leaves. The miRNA prediction was processed as described by Yang and Li (2011). Grapevine Pinot Noir genome (http://bowtie-bio.sourceforge.net/) was used as reference sequences.

Putative mature miRNA sequences were used as queries to search against the grape gene (http://www.genoscope.cns.fr/) using online server psRNATarget (Dai and Zhao, 2011). Alignments between each miRNA and its target(s) should satisfy the following criteria: the maximum expectation is 3.0; the length for complementarity scoring is $20 \mathrm{bp}$; target accessibilitymaximum energy to unpair the target site (UPE) is no more than 25.0; upstream and downstream flanking length around the target site were 17 and 13 respectively; range of central mismatch leading to translational inhibition was 9-11 nucleotides. Further, GO analysis was carried out for target genes by AgriGO (http://bioinfo.cau.edu.cn/agriGO/) (Zhou et al., 2010).

\section{Expression Pattern Analysis of miRNAs}

In this study, semi-quantitative RT-PCR and stem-loop realtime PCR were employed to display the expression patterns of vvi-miRNAs with 5.8SrRNA as reference gene. The semiquantitative RT-PCR shared the same primers with stemloop real-time PCR. The primers were designed following the instructions of Chen (Chen et al., 2005). The $5^{\prime}$ end of reverse transcription primers (RT primers) was universal, while the $3^{\prime}$ end was specific by reverse complement with 7-10 bases from the $3^{\prime}$ end of the mature miRNA. We used Oligo(dt) to reverse transcribe 5.8SrRNA. The forward quantitative primers were similar to mature miRNA sequences with about 9 bases excluded at their $3^{\prime}$ ends and 4-6 bases added at the $5^{\prime}$ ends according to the $\mathrm{G} / \mathrm{C}$ content. Meanwhile, the universal reverse primer was a part of stem-loop RT primers. All primers were synthesized by the Invitrogen company (Table S1). Total RNAs for expression pattern analysis were extracted from grapevine leaves inoculated with $E$. necator for $0,12,24,48,72,96,120$, and $144 \mathrm{~h}$ as previously described.
The reverse transcription of the vvi-miRNA was done as follows: prepare reaction mixture of $1.0 \mu \mathrm{g}$ total RNA, $1.0 \mu \mathrm{l}$ RT primer $(10 \mu \mathrm{M})$, and $1.0 \mu \mathrm{l}$ Oligo(dT) $(10 \mu \mathrm{M})$ in a RNAsefree PCR tube with RNAse-free water added to a total volume of $10.0 \mu \mathrm{l}$. The mixture was then incubated at $80^{\circ} \mathrm{C}$ for $5 \mathrm{~min}$ followed by cooling on ice for $10 \mathrm{~min}$. Then, $10.0 \mu \mathrm{l}$ mixture (4 $\mu 15 \times$ M-MLV buffer (Takara), $2.5 \mu \mathrm{l}$ dNTPs (10 mM each, Takara), $0.5 \mu \mathrm{l}$ RNAse inhibitor $(40 \mathrm{U} / \mu \mathrm{l})$ and $1 \mu \mathrm{l}$ Rtase M-

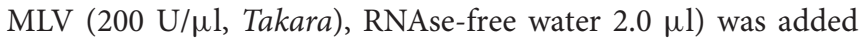
to the same PCR tube. The $20.0 \mu \mathrm{l}$ reactions were incubated at $16^{\circ} \mathrm{C}$ for $30 \mathrm{~min}$, followed by 60 cycles of $20^{\circ} \mathrm{C}$ for $30 \mathrm{~s}, 42^{\circ} \mathrm{C}$ for $30 \mathrm{~s}$ and $50^{\circ} \mathrm{C}$ for $1 \mathrm{~s}$ and finally inactivated the reaction at $85^{\circ} \mathrm{C}$ for $5 \mathrm{~min}$.

Semi-quantitative RT-PCR reactions for vvi-miRNAs were performed for $4 \mathrm{~min}$ at $95^{\circ} \mathrm{C}$, followed by 30-40 cycles (adjusted for individual vvi-miRNA) of $94^{\circ} \mathrm{C}$ for $30 \mathrm{~s}, 56^{\circ} \mathrm{C}$ for $30 \mathrm{~s}, 72^{\circ} \mathrm{C}$ for $30 \mathrm{~s}$ and final elongation at $72^{\circ} \mathrm{C}$ for $5 \mathrm{~min}$. The reaction mixture was $2 \times$ rTaq $10 \mu \mathrm{l}$, cDNA $1.0 \mu \mathrm{l}$, forward primer $(10 \mu \mathrm{M}) 1.0 \mu \mathrm{l}$, reverse primer $(10 \mu \mathrm{M}) 1.0 \mu \mathrm{l}, \mathrm{ddH}_{2} 0$ up to $20 \mu l$.

Real-time quantitative PCR was carried out using a SYBR premix Ex TaqII kit protocol on an iQ5_Real-Time System (BioRad). The $21 \mu \mathrm{l}$ PCR included $2.0 \mu \mathrm{l}$ RT product, $10 \mu \mathrm{l} 2 \mathrm{xSYBR}$ Taq, $0.8 \mu \mathrm{l}$ forward primer $(10 \mu \mathrm{M}), 0.8 \mu \mathrm{l}$ reverse primer $(10$ $\mu \mathrm{M})$ and $7.4 \mu \mathrm{l}$ RNAse-free $\mathrm{H}_{2} \mathrm{O}$. The reactions were incubated in a 96-well plate for $3 \mathrm{~min}$ at $95^{\circ} \mathrm{C}$, followed by 45 cycles of $95^{\circ} \mathrm{C}$ for $5 \mathrm{~s}$ and $58^{\circ} \mathrm{C}$ for $30 \mathrm{~s}$. Relative expression was calculated using the $2^{-\Delta \Delta \mathrm{Ct}}$ method, normalized to the expression of $5.8 \mathrm{~S}$ rRNA. Three biological and technical replicates were used for each sample.

\section{Expression Analysis of miRNA Targets}

The expression of some target genes was analyzed by realtime quantitative PCR. Primers are listed in Table S1. Total RNAs were extracted from "Baihe-35-1" leaves with the same inoculation treatment as previously described. First-strand cDNA was synthesized from $1.0 \mu \mathrm{g}$ DNase-treated total RNA according to the instruction of PrimeScript II 1st strand kit (Takara). qRT-PCR was carried out on an IQ5 real-time PCR machine (Bio-Rad, Hercules, CA) using SYBR green (Takara Biotechnology), according to the protocol provided. The relative expression was calculated using the $2^{-\Delta \Delta \mathrm{Ct}}$ method normalized to the expression of $5.8 \mathrm{~S}$ rRNA.

\section{Construction of Transient Expression Vectors}

Pro35s:MIR2118 and Pro35s: $\triangle R P P 13-G U S$ vectors were constructed to verify interaction between vvi-NewmiR2118 and its target RPP13 (Recognition of Peronospora Parasitica, RPP). MIR2118 was PCR amplified from "Baihe-35-1" genomic DNA with the primer combinations $5^{\prime}$-gggGAGCTCCTA GGGTTTTTGCAGAGTAGTATAAAAGG-3' and $5^{\prime}$-gggGGT ACCGTAG CTTCAAGTTCAAGTCAAATCGAAC- $3^{\prime}$. Then MIR2118 was cloned into vector pCAMBIA1307 harboring the $35 S$ promoter. For the Pro $35 \mathrm{~s}: \triangle R P P 13-G U S$ construct, 998bp coding sequence from the start codon of "Baihe-35-1" RPP13 cDNA was amplified using the primer pair $5^{\prime}$-GGATCCATG 
GCTTCTTGG-3' and 5'-GAATTCGTCGGATGTGCACAG GTGTCT-3', termed $\triangle$ RPP13. This includes the interaction regions of vvi-NewmiR2118 and RPP13. $\triangle \mathrm{RPP} 13$ was then inserted into vector pCAMBIA0390, previously modified by our team by inserting coding sequences of GUS after its $35 \mathrm{~S}$ promoter. All constructs were confirmed with standard sequencing before the correct construct was transformed into Agrobacterium strain GV3101 by electroporation using Eppendorf 4308.

\section{Nicotiana benthamiana Transient Transformation}

For transient analysis of the To verify the interaction of vviNewmiR2118 and $\triangle$ RPP13, we transiently expressed them through expression vectors constructed above in Nicotiana benthamiana by Agrobacterium tumefaciens transformation. Plants used grew in a growth chamber at $22^{\circ} \mathrm{C}$ under $16 \mathrm{~h}$ light (Sparkes et al., 2006) for 6 weeks. The healthy third and fourth leaves from the apical meristem were infiltrated by $1 \mathrm{ml}$ needleless syringe filled with Agrobacterium tumefaciens $\left(\mathrm{OD}_{600}=0.6\right)$ or the control suspension throughout the whole abaxial surface. The transformation were settled as: (A) penetration buffer, (B) Pro $35 s: M I R 2118$, (C) Pro $35 s: G U S$, (D) Pro $_{35 s}: \triangle R P P 13-G U S$, (E) Pro $35 s: M I R 2118$ and Pro $35 s: G U S$ $(\mathrm{v} / \mathrm{v}=5: 1)$, and $(\mathrm{F})$ Pro $_{35 S}: M I R 2118$ and Pro $355: \triangle R P P 13-$ GUS $(\mathrm{v} / \mathrm{v}=5: 1) \quad$ co-transformation (Figure 10). The infiltrated leaves were marked before the whole plants were replaced into the growth chamber and cultivated under previous growing conditions before they were collected after $48 \mathrm{~h}$ infiltration for GUS staining and fluorescence quantitative assay performed as described by Bradford (1976) and Jefferson et al. (1987). Three biological repeats were performed.

\section{RESULTS}

\section{Small Rans from "Baihe-35-1"}

To identify miRNAs from "Baihe-35-1," total RNAs from young leaves inoculated with E. necator (see Materials and Methods) and the control were extracted separately for high throughput sequencing, yielding 24839978 and 25714911 raw read totals from the control $(\mathrm{BH}-\mathrm{C})$ and treated $(\mathrm{BH}-\mathrm{T})$ libraries, respectively (Table 1). After filtration, 24829158 and 25703809

TABLE 1 | Results of small RNA high-throughput sequencing for leaves of Vitis pseudoreticulata cv "Baihe-35-1."

\begin{tabular}{llrlr}
\hline & \multicolumn{2}{c}{ BH-C } & \multicolumn{2}{c}{ BH-T } \\
\hline Total reads & 24839978 & $100.00 \%$ & 25714911 & $100.00 \%$ \\
Clean reads & 24829158 & $99.96 \%$ & 25703809 & $99.96 \%$ \\
Unique reads & 3964135 & $15.96 \%$ & 3777370 & $14.69 \%$ \\
Mapped reads & 1626820 & $6.55 \%$ & 1584520 & $6.16 \%$
\end{tabular}

Total reads, clean reads, unique reads, and mapped reads are referenced to the number of all reads, reads removed of low quality reads, reads getting rid of redundancy and reads mapped on grape genome respectively in each group. $\mathrm{BH}-\mathrm{CK}$ referred to the control group and $\mathrm{BH}-\mathrm{T}$ the experimental group. clean reads were obtained, which merged into 3964135 and 3777370 unique reads from $\mathrm{BH}-\mathrm{C}$ and $\mathrm{BH}-\mathrm{T}$ libraries. Finally 1626820 reads (41.04\% of unique reads) in the $\mathrm{BH}-\mathrm{C}$ and 1584520 reads ( $41.95 \%$ of unique reads) in the $\mathrm{BH}-\mathrm{T}$ were mapped onto the vitis reference genome, which occupied $6.55 \%$ and $6.16 \%$ of clean reads, respectively. Length distribution analyses showed that the small RNA length from $V$. pseudoreticulata varied from 18-nt to 27-nt in both the control and the treated group, with two main peaks at 21-nt and 24-nt (Figure 1).

\section{vvi-miRNA Identification from "Baihe-35-1"}

Clean reads from high-throughput sequencing were processed according to miRDeep-P workflow with the $V$. vinifera genome as the reference sequence. There were 250 miRNAs, with 126 known miRNAs and 124 candidates (named vvi-New) (Table S2) identified from Chinese wild V. pseudoreticulata. The known miRNAs were classified into 37 families. In our research, 38 known miRNAs (labeled as vvi-NewmiRNA; Table S2) were identical to, or had limited nucleotide shifts from previously reported miRNAs in mature sequences, but were found different stem-loop precursors. Moreover, most known miRNAs from Chinese wild grape were detected in both libraries with 17 detected only in the control, and 15 specific in the treated group (Table S3). Oppositely, most candidates could only be detected from one library (Table S3). However, the read numbers of the candidates were much lower than those of the known miRNAs. Members in different families varied greatly. The largest families were vvi-miR395 and vvi-miR535 with 13 members, followed by vvi-miR156 with 8 members and vvimiR169 and vvi-miR171 with 7 members each, while seven families (vvi-miR159, vvi-miR162, vvi-miR164, vvi-miR390, vvi-miR397, vvi-miR399, vvi-miR408) only had one member detected. Based on our sequencing, there were significant differences in the expression levels of known miRNAs. The read numbers of most miRNAs were several hundreds. But the expressions of vvi-miR156f,g, vvi-miR166f,g, vvi-miR167b,e,d, vvi-miR3636, and vvi-miR535b,c were extremely high. While several miRNA families or members such as miR156a, miR169d,j, and miR395 had low expression with less than 100 reads detected.

The length of miRNA mature sequences from "Baihe-35-1" were distributed among 18-24 nucleotides. Though most vvi-miRNAs were 21 or 22 -nt, the longest was vvi-miR393b with 24 nucleotides and the shortest was 18-nt vvi-miR396a. Family members could be sequenced with different lengths; for example, members of the family miR156 had lengths of 20 and 21-nt, miR167 had lengths of 21 and 22-nt, miR169 and 398 had lengths of 21 and 23-nt.

Figure 2 shows the nucleotide bias at each position in vvi-miRNAs. In agreement with previous reports, $U$ was dominant at the first position of vvi-miRNAs from Chinese wild $V$. pseudoreticulata, which is a well-established characteristic of miRNAs (Carra et al., 2009), but C was the most frequently used base (about 38.6\%). At almost every site of mature vvi-miRNA sequences, there was bias for a specific ribonucleotide among A, U, C, and G. The phenomenon that most A and $\mathrm{U}$ were distributed in the front $5^{\prime}$ end of a sequence with $\mathrm{G}$ 


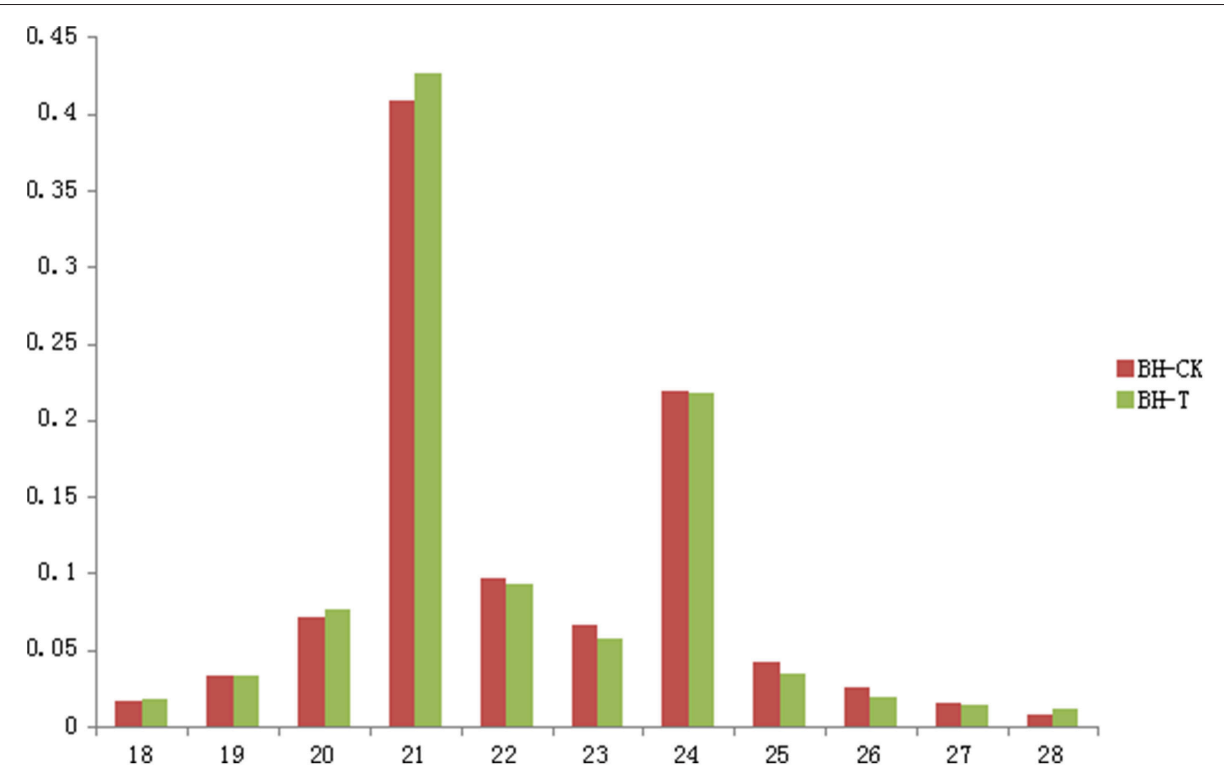

FIGURE 1 | The length distribution of small RNA sequences in leaves inoculated with Erysiphe necator and the control. The X-axis shows nucleotide numbers of small RNA sequences while the $y$-axis shows the frequency of reads.

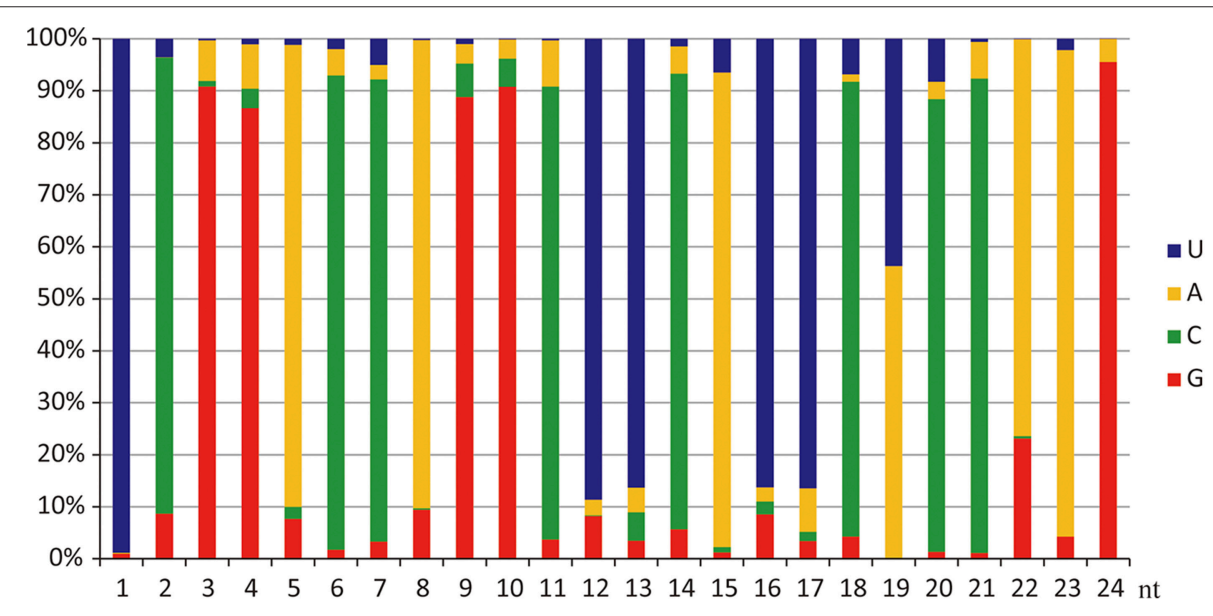

FIGURE 2 | vvi-miRNA nucleotide bias at each position. $U$ was dominant at the first position of $5^{\prime}$ end, while $\mathrm{C}$ was the most frequently used base in miRNAs of Chinese wild Vitis pseudoreticulata. Each site had its favorite ribonucleotide among A, U, C, and G.

and $\mathrm{C}$ more frequent in the 6-18 positions may have some relationship with miRNA function mechanisms, such as binding with corresponding targets (Xu et al., 2010).

\section{Conserved and Non-conserved miRNAs in "Baihe-35-1"}

One of the notable characteristics of plant miRNA is its highly-conserved nature. This may be partially explained by its involvement in biological development and stress responses. Unsurprisingly, almost all of the 24 conserved miRNA families (Jones-Rhoades et al., 2006; Rajagopalan et al., 2006) but vvimiR161, vvi-miR168, vvi-miR391 and vvi-miR394 were detected in our libraries (Table S4). This indicates that the quality of our sRNA high-throughput sequencing is good, and the tools chosen for miRNA identification are also suitable.

In addition to conserved miRNAs, 15 known non-conserved miRNA families were also identified in wild Chinese grape "Baihe-35-1". Alabi et al. (2012) found seven miRNA families vvi-miR472, vvi-miR529, vvi-miR530, vvi-miR827, vvi-miR894, vvi-miR1507, and vvi-miR1511 that had not been reported previously from grapevine. However, only vvi-miR529, vimiR530 and vvi-miR827 were detected by us in wild Chinese V. pseudoreticulata. But we found that vvi-NewmiR2118 was highly expressed with 37338 reads in the control and 25426 reads in the inoculated samples. 


\section{Target Prediction of vvi-miRNAs from "Baihe-35-1"}

To elucidate the function of miRNAs, it is essential to identify and characterize their targets. We employed the tool psRNATarget (http://plantgrn.noble.org/psRNATarget/) to identify the potential targets of all Chinese wild $V$. pseudoreticulata miRNAs. Finally a total of 485 experimental verified or putative transcripts were predicted for 214 vvi-miRNAs. Nine known miRNAs (vvi-miR399a, vvi-miR399h, vvi-miR396a, vvi-NewmiR403a, vvi-miR2111, vvi-miR2950, vvi-NewmiR4376, vvi-NewmiR399, vvi-NewmiR403b) and 24 candidates had no target mRNA predicted. Consistent with previous reports, many targets of conserved miRNA were transcription factors. For example, vvi-miR160 mainly targeted $A R F \mathrm{~s}$, vvi-miR171 targeted several members of the SCL (scarecrow-like) family. As reported by Carra et al. (2009), in our research, the mature sequence alignment (Figure 3) showed high similarity between vvimiR535 and vvi-miR156 family from Chinese wild grape. This

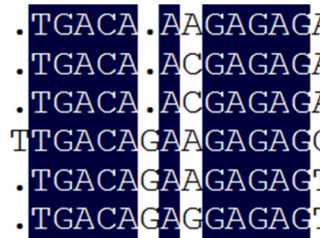

AGAGCACA
AGAGCATG
AGAGCACG
GGAGCAC.
IGAGCAC.
IGAGCAC.

FIGURE 3 | Sequence alignment of miR535 and miR156 from "Baihe-35-1." The result revealed high similarity between vvi-miR156 and vvi-miR535 family in Viti, indicating possible evolutional and functional relationships. Mature miRNAs are shown in $5^{\prime}$ to $3^{\prime}$ orientation. Dark blue shading indicates nucleotides were conserved in $100 \%$ of the sequences. explains their same target prediction-the transcription factor SPL (squamosa promoter-binding-like protein). In contrast to conserved miRNAs, targets of non-conserved vvi-miRNAs had wider diversity. This indicates that it"s more likely that non-conserved miRNAs are functionally species specific.

Gene Ontology (GO) analysis (Figure 4) was carried out for all putative targets. According to the GO term identification, the molecular function involved mainly in binding, catalytic, transcription regulation, transporter, and molecular transducer activity. For the biological process, the putative target transcripts of miRNAs were classified into 23 categories, and involved mainly in growth and development, multicellular organismal process, metabolism and stimulus response. Based on GO terms and previous studies on $\mathrm{R}$ genes and genes related to defense responses, 49 disease resistance genes being targeted by 32 vvimiRNA families (15 known and 17 candidates) were identified in our research. Remarkably, several miRNA families, including vvimiR156, 159, 171, 172, 390, and 396, traditionally regulating the growth and development of plants were found with R or R-related gene targets.

\section{Expression and Identification of miRNAs Involved in Powdery Mildew Resistance}

When plants suffer environmental stresses, the expression of the miRNA repertoire often shows fluctuation. Here, the expression of miRNAs was standardized by RPKM (reads per kilo bases per million reads). We found 131 miRNAs were up-regulated and 119 were down-regulated after inoculation with E. necator. In our study, 32 vvi-miRNAs corresponding to 68 non-redundant sequences, displayed significant differential expressions (fold change $\geq 2$ and $P<0.5$ ), including 18 up-regulated and 11

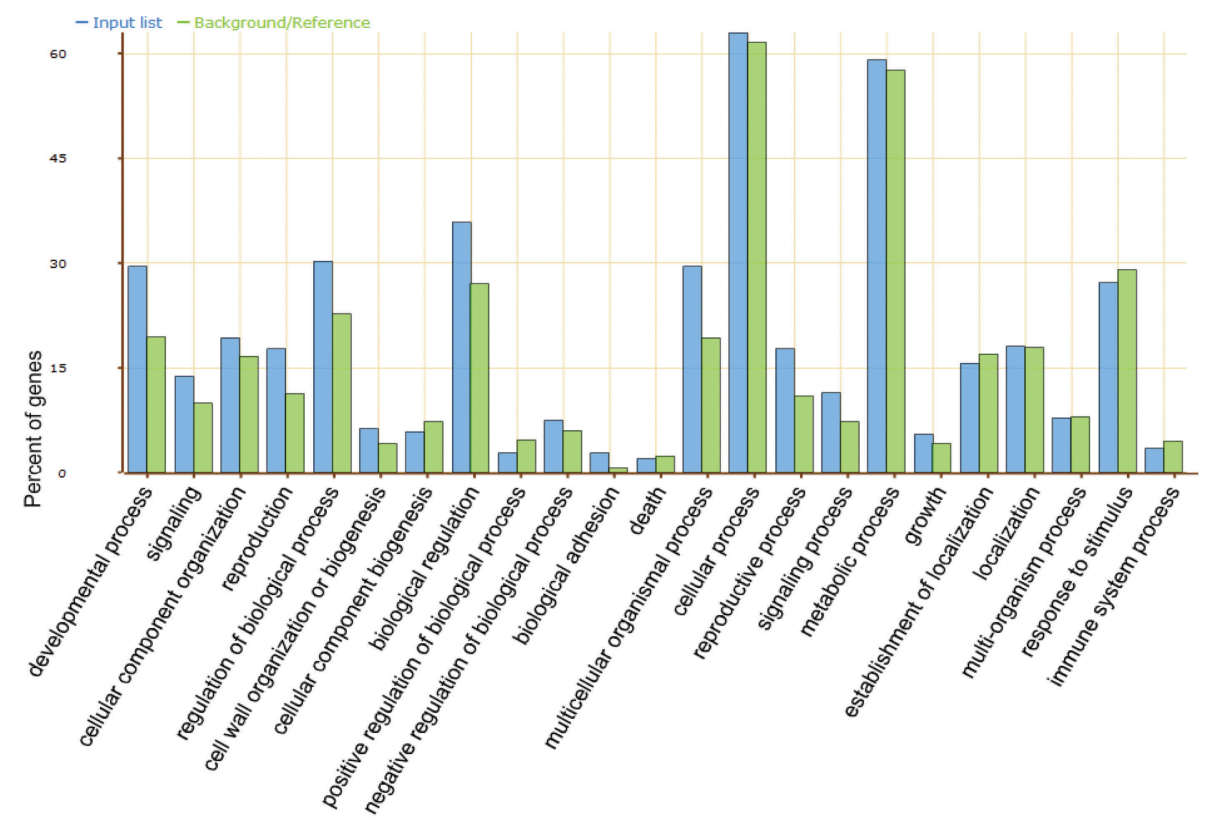

FIGURE 4 | GO annotation for all the predicted targets of miRNAs got from "Baihe-35-1." 
down-regulated known miRNAs and 3 up-regulated candidates (vvi-New27, vvi-New 69a and vvi-New82; Table S5). Meanwhile, vvi-miR3636, vvi-miR3623, and vvi-New27 were only found in the experiment group, so their expression may require induction by pathogens. In addition, the expression of vvi-miR396b showed the largest rise, followed by vvi-miR164d and vvi-miR172d. The expression level of vvi-miR479 declined most, to $9 \%$ of normal level after inoculation with $E$. necator. Other significantly reduced miRNAs were vvi-MIR3633a, vvi-NewmiR535a, and vvi-NewmiR827. Vvi-MIR156a, 166a, 167c, vvi-NewmiR403a,b failed to be detected in the treated library.

To verify our identification of miRNAs from wild Chinese $V$. pseudoreticulata and uncover their expression conditions, we performed semi-quantitative RT-PCR for candidate vvi-New27, conserved vvi-miR159c, 396b, 172d, 171b and non-conserved vvi-NewmiR535, NewmiR482, and NewmiR2118 (Figure 5). Before E. necator inoculation, vvi-miR396b, vvi-NewmiR2118 and vvi-NewmiR482 expressed strongly in leaves of "Baihe-35-1," vvi-miR171b and vvi-New27 had obvious expression while vvimiR159c, vvi-NewmiR535 and miR172d expressed weakly. After E. necator inoculation, the expressions of vvi-miR396b, vviNewmiR482, vvi-NewmiR535 and vvi-New27 did not change markedly, while vvi-NewmiR2118 and vvi-miR171b reduced sharply after 96 h, vvi-miR172d was down-regulated after 48 h, the expression of vvi-NewmiR535 was up-regulated soon after inoculation for $48 \mathrm{~h}$ and such high expression lasted for 3 days before reducing rapidly to its normal level by about 120 $\mathrm{h}$ after inoculation. The expression patterns of vvi-miR172d, vvi-NewmiR2118, vvi-NewmiR482, vvi-miR159c, vvi-New27,

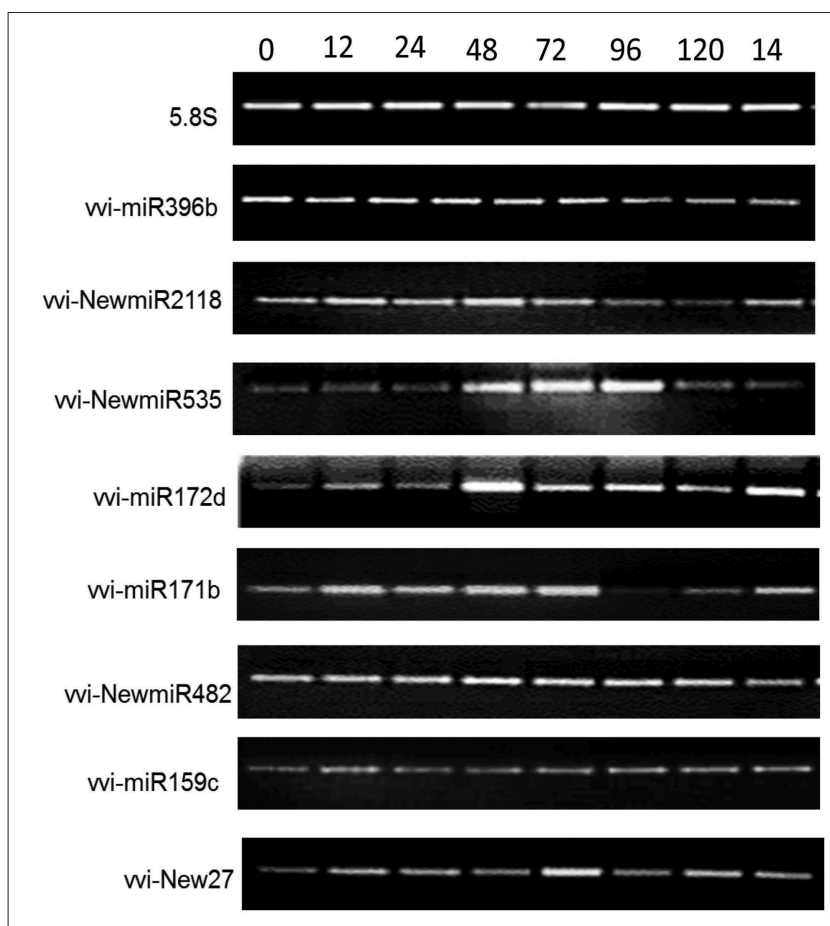

FIGURE 5 | Expression analysis by semi-quantitative RT-PCR of miRNA from "Baihe-35-1" after inoculation by Erysiphe necator for 0, 12, 24,

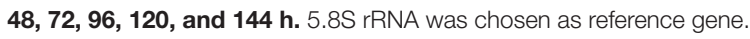

and vvi-NewmiR535 analyzed by semi-quantitative RT-PCR and miRDeep-P were consistent but for vvi-miR396b and vvimiR171b.

Apart from semi-quantitative RT-PCR, real-time qRT-PCR (qRT-PCR) was also done for several miRNAs and their targets (Figure 6). The expression of vvi-miR171b, vvi-NewmiR2118, vvi-NewmiR535, and vvi-New27 in Chinese wild grape was reduced noticeably after pathogen infection, with the main reduction occurring $24-72 \mathrm{~h}$ after inoculation. The accumulation of vvi-miR396 decreased markedly $12 \mathrm{~h}$ after inoculation but then gradually recovered to its normal level. Vvi-NewmiR2118 was fast and markedly down-regulated during after inoculated $24 \mathrm{~h}$ in Baihe-35-1, while in susceptible grapevine Piont Noir its expression did not show obvious changes. So we suggested the involvement of vvi-NewmiR2118 from $V$. pseudoreticulata in resistance to powdery mildew. The expressions of vvi-NewmiR482 and vvi-miR159c fluctuated gently after pathogen infection. Except vvi-miR396b, vvi-NewmiR535, and vvi-New27, the expression patterns of most miRNAs examined by semi-quantitative RT-PCR and qRT-PCR were similar.

Since plant miRNA negatively regulates its target(s), qRTPCR was also carried out for some target genes (Figure 6). Results displayed a negative relationship between vvimiR171b and target SCL6 (GSVIVT0102768000, scarecrow-like protein 6), which also applied to vvi-miR159c and its target GAMYB-like (GSVIVT01012447001); vvi-miR396b and its two targets-GRF9 (GSVIVT01027535001, growth-regulating factor 9-like) and leucine aminopeptidase chloroplastic-like (GSVIVT01023604001) expressed stably except at some individual test points. The expression of vvi-NewmiR535 and target SPL9 (GSVIVT01033519001, squamosa promoter binding protein 9-like) did not show clear relation. However, there was obvious opposite trend in the expression changes of vvi-NewmiR2118 and its two targets: disease resistance protein at3g14460-like (GSVIVT01001446001) and disease resistance protein RPP13-like (GSVIVT01013307001).

Based on the differential expression and GO term(s) of target gene(s), 13 known vvi-miRNA families and one candidate vviNew69a with 36 targets were identified as miRNAs that may be involved in enhanced powdery mildew resistance in wild Chinese $V$. pseudoreticulata (Table 2). And their targets were either $\mathrm{R}$ genes or signal-related genes involved in biotic stress.

\section{Characteristics of vvi-NewmiR2118 and Its Targets}

Among the miRNAs from wild Chinese V. pseudoreticulata predicted to be involved in powdery mildew resistance, vviNewmiR2118 was most interesting. Its expression decreased quickly and strongly in infected leaves and its predicted targets were all NBS-LRR type R genes. In "Baihe-35-1," the gene MIR2118 was located on chromosome 19 and its precursor sequence was located from 9739640 to 9739711 . The reads of vvi-NewmiR2118 showed significant accumulation on the gene GSVIVT01014787001, providing reliable information about the transcription and process of the precursor of miR2118 (Figure 7). 


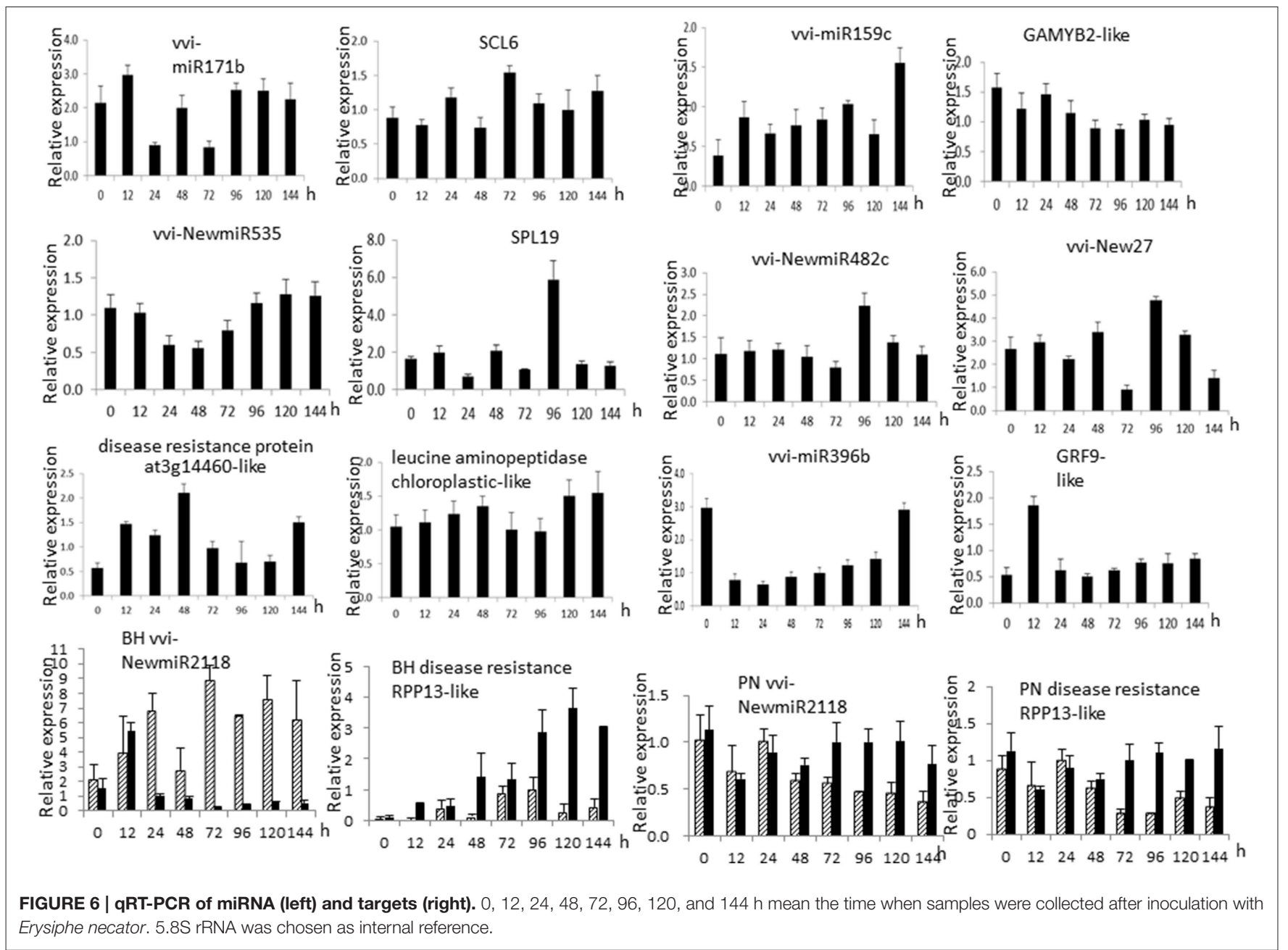

The conservative characteristics of miR2118 were also analyzed by blasting sequences of miR2118 from 13 species (Figure 8A). The alignment result indicated that miR2118 is pretty conserved in plants except frequent base changes at the third and ninth position from the $5^{\prime}$ end. The bases at the eleventh and sixteenth position (according to vvi-NewmiR2118) were almost fixed with $\mathrm{C}$ and $\mathrm{A}$, respectively. Multiple alignment of vvi-NewmiR2118 and all 47 published miR2118 sequences from miRBase 21.0 (Figure 8B) showed that miR2118 from annual plants such as Zea mays, Oryza sativa L. and Aegilops tauschii were closely related, while miRNAs from perennial plants were clustered. Particularly, vvi-NewmiR2118 shared sequence identity with Vigna unguiculata, Phaseolus vulgaris, and Panax ginseng.

We predicted eight targets for vvi-NewmiR2118. Among them, five were NBS-LRR disease resistance genes containing NB-ARC and LRR domains (GSVIVT01001446001putative disease resistance protein At3g14460-like; GSVIVT01007026001 putative disease resistance protein At3g14460-like; GSVIVT01014595001 disease resistance protein At4g27190-like; GSVIVT01028627001 disease resistance protein RPM1like; GSVIVT01013307001 disease resistance RPP13-like protein1) and one was the TMV resistance protein N-like (GSVIVT01029009001). The 3' end of vvi-NewmiR2118 bound with the conserved p-loop motif of all targets, except the disease resistance protein RPM1-like (Figure 9).

\section{Verification of the Interaction between vvi-NewmiR2118 and RPP13}

Disease resistance gene RPP13 (Recognition of Peronospora parasitica, RPP) was one of the predicted targets of vviNewmiR2118, whose expression could be inhibited by degrading the mRNA. We transiently co-expressed vvi-NewmiR2118 and $\triangle \mathrm{RPP} 13-\mathrm{GUS}$ in tobacco leaves and verified their interaction by histochemical staining and GUS fluorescence quantitative assay.

Based on histochemical staining (Figure 10), GUS expressed strongly when transformed alone into tobacco leaves (C,D). This also happened in leaves co-transformed by Pro $_{35 S}$ :MIR2118 and Pro $_{35 S}: G U S(\mathrm{v} / \mathrm{v}=5: 1)$ (E) because vvi-NewmiR2118 could not silence GUS due to the deficiency of interaction site. But in leaves co-transformed by Pro $_{35 S}: M I R 2118$ and Pro $355: \triangle R P P 13-$ GUS (v/v = 5:1) $(\mathrm{F})$, only very weak GUS expression was found. The explanation was that $\triangle R P P 13$ introduced target sequence 
TABLE 2 | vvi-miRNAs involved in powdery mildew resistance of wild Chinese Vitis pseudoreticulata.

\begin{tabular}{|c|c|c|}
\hline vvi-miR & Target & Target desc. \\
\hline vvi-MIR156f & GSVIVT01016962001 & $\begin{array}{l}\text { Probable disease resistance protein } \\
\text { at5g63020-like }\end{array}$ \\
\hline \multirow[t]{3}{*}{ vvi-MIR159c } & GSVIVT01010961001 & Zinc finger protein MAGPIE-like \\
\hline & GSVIVT01037667001 & r2r3-myb transcription \\
\hline & GSVIVT01001155001 & Copalyl diphosphate synthase \\
\hline vvi-MIR164d & GSVIVT01016290001 & Para-aminobenzoate synthase-like \\
\hline vvi-MIR166a & GSVIVT01034812001 & TMV resistance protein $\mathrm{n}$-like \\
\hline vvi-MIR166f & GSVIVT01034812001 & TMV resistance protein $\mathrm{n}$-like \\
\hline \multirow[t]{2}{*}{ vvi-MIR172d } & GSVIVT01016352001 & $\begin{array}{l}\text { Ethylene-responsive transcription } \\
\text { factor rap2-7-like }\end{array}$ \\
\hline & GSVIVT01025100001 & $\begin{array}{l}\text { Ethylene-responsive transcription } \\
\text { factor rap2-7-like }\end{array}$ \\
\hline vvi-MIR3633a & GSVIVT01023557001 & $\begin{array}{l}\text { Disease resistance protein } \\
\text { at4g27190-like }\end{array}$ \\
\hline \multirow[t]{2}{*}{ vvi-MIR390 } & GSVIVT01015035001 & Auxin response factor 19 -like \\
\hline & GSVIVT01038628001 & $\begin{array}{l}\text { Leucine rich repeat family } \\
\text { expressed }\end{array}$ \\
\hline \multirow[t]{2}{*}{ vvi-MIR396b } & GSVIVT01035367001 & $\begin{array}{l}\text { Receptor-like protein kinase } \\
\text { hsl1-like }\end{array}$ \\
\hline & GSVIVT01037219001 & TMV resistance protein n-like \\
\hline vvi-MIR479 & GSVIVT01035459001 & Transcription repressor myb4 \\
\hline vvi-New69a & GSVIVT01003955001 & $\begin{array}{l}\text { 1-deoxy-d-xylulose 5-phosphate } \\
\text { synthase }\end{array}$ \\
\hline \multirow[t]{6}{*}{ vvi-NewmiR2118 } & GSVIVT01001446001 & $\begin{array}{l}\text { Disease resistance protein } \\
\text { at3g14460-like }\end{array}$ \\
\hline & GSVIVT01007026001 & $\begin{array}{l}\text { Disease resistance protein } \\
\text { at3g14460-like }\end{array}$ \\
\hline & GSVIVT01013307001 & $\begin{array}{l}\text { Disease resistance } \triangle \mathrm{RPP} 13 \text {-like } \\
\text { protein 1-like }\end{array}$ \\
\hline & GSVIVT01014595001 & $\begin{array}{l}\text { Disease resistance protein } \\
\text { at } 4 \text { g27190-like }\end{array}$ \\
\hline & GSVIVT01028627001 & $\begin{array}{l}\text { Disease resistance protein } \\
\text { rpm1-like }\end{array}$ \\
\hline & GSVIVT01029009001 & TMV resistance protein n-like \\
\hline vvi-NewmiR482 & GSVIVT01033046001 & Della protein gai1-like \\
\hline vvi-NewmiR535a & GSVIVT01011939001 & $\begin{array}{l}\text { Mediator of rna polymerase ii } \\
\text { transcription subunit } 25 \text {-like }\end{array}$ \\
\hline
\end{tabular}

Each list from left to right represented miRNAs involved in powdery mildew resistance from "Baihe-35-1," targets relative to defense or resistance responses and their description.

which could be identified and cleaved by vvi-NewmiR2118, which as a result blocked the expression of GUS. So GUS staining verified the negative regulation of vvi-NewmiR2118 to $\triangle$ RPP13.

The differences in GUS protein activity in each treatment were further analyzed by fluorescence quantitative assay. There were no significant difference in GUS activity among the control and the combination of Pro $_{35 S}$ :MIR2118 and Pro $_{35 s}: \triangle R P P 13-G U S$. This also occurred with leaves transformed by Pro $_{35 S}$ :GUS, Pro $_{35 S}: \triangle R P P 13-G U S$ and leaves co-transformed by Pro $_{35 S}$ :MIR2118 and Pro $_{355}$ :GUS. However, the GUS activity was significantly different between the two groups. So the GUS fluorescence quantitative assay supported the results of histochemical staining. Based on these series of experiments, we believed that RPP13 was a real target of vvi-NewmiR2118.

\section{DISCUSSION}

Small RNA high-throughput sequencing is now a widelyused method for miRNA screening, which has resulted in the discovery of a huge number of sRNA from a wide range of plant species. Therefore in this study, we also adopted this method to identify miRNAs in Chinese wild V. pseudoreticulata. The common lengths of plant sRNA are usually 21 or $24 \mathrm{nt}$ (Rajagopalan et al., 2006; Fahlgren et al., 2007; Moxon et al., 2008). In many plants, the number of $24 n t$ sRNA usually exceed 21nt sRNA, for example, in gymnosperms Taxus chinensis (Qiu et al., 2009), in monocots Oryza sativa (Morin et al., 2008) and in eudicots Arabidopsis (Rajagopalan et al., 2006), tomato (Moxon et al., 2008), and Citrus trifoliata (Song et al., 2010). But according to our results, the $21 \mathrm{nt}$ sRNAs were more abundant than the $24 \mathrm{nt}$ ones in the leaf tissue of wild Chinese V. pseudoreticulata, which contrasts with many other plants. In other grapevine species Populus balsamifer (Barakat et al., 2007), Pinus cordata (Morin et al., 2008) and in leaves, berries, tendrils and flowers of Pinot Noir (Pantaleo et al., 2010), the 21nt class of sRNAs also showed higher abundance. Since 21 and $24 \mathrm{nt}$ are the typical lengths of miRNA and siRNA, respectively, we suggest the type of sRNA playing dominant roles in plant species may be different, with annuals more generally having 24nt miRNA and siRNA, and perennials 21 nt miRNA.

MiRDeep-P is an effective and efficient tool for plant miRNA identification. In this research, miRDeep-P combined with sRNA high-throughput sequencing not only detected known vvi-miRNAs, but also predicted 124 candidate miRNAs from leaves of "Baihe-35-1" under E. necator inoculation condition. Previous studies have reported several novel miRNAs from the $V$. vinifera grapevine cultivars Corvina and Pinot Noir respectively (Mica et al., 2009; Pantaleo et al., 2010). Meanwhile, another study describes new miRNAs from the interspecific table grape cultivar Summer Black (Wang et al., 2011), thus enlarging the vvi-miRNA repertoire. However, not all these reported novel miRNAs were found from "Baihe-35-1." This may be due to the tissue and stress condition we used to construct the libraries. The different genetic backgrounds of the wild Chinese $V$. pseudoreticulata, Pinot Noir and Corvina could also explain this phenomenon. The $3^{\prime}$ ends of mature sequences in some miRNA families, including vvi-miR167b/c/d, vvi-miR171b/e and vvi-miR396a/b/c, contain one to three bases variation. We think this is possibly caused by inaccurate cleavage of DCL1 to miRNA precursors.

An interesting finding in our research is the high sequence similarity between vvi-miR156 and vvi-NewmiR535. Since the conserved miRNA evolved earlier than the non-conserved, we suggest that vvi-miR156 may be the ancestor of vvi-NewmiR535 and form a superfamily. However, many studies prove that the SPL family is mainly regulated by miR156 (Xie et al., 2006), indicating that non-conserved miRNAs have evolved new and different functions from their ancestors.

In this research, a large number of target genes have been predicted for miRNAs. In terms of conserved miRNAs, their targets were mainly transcription factors, which agrees with previous studies. The origin of plant miRNA dates back 400 


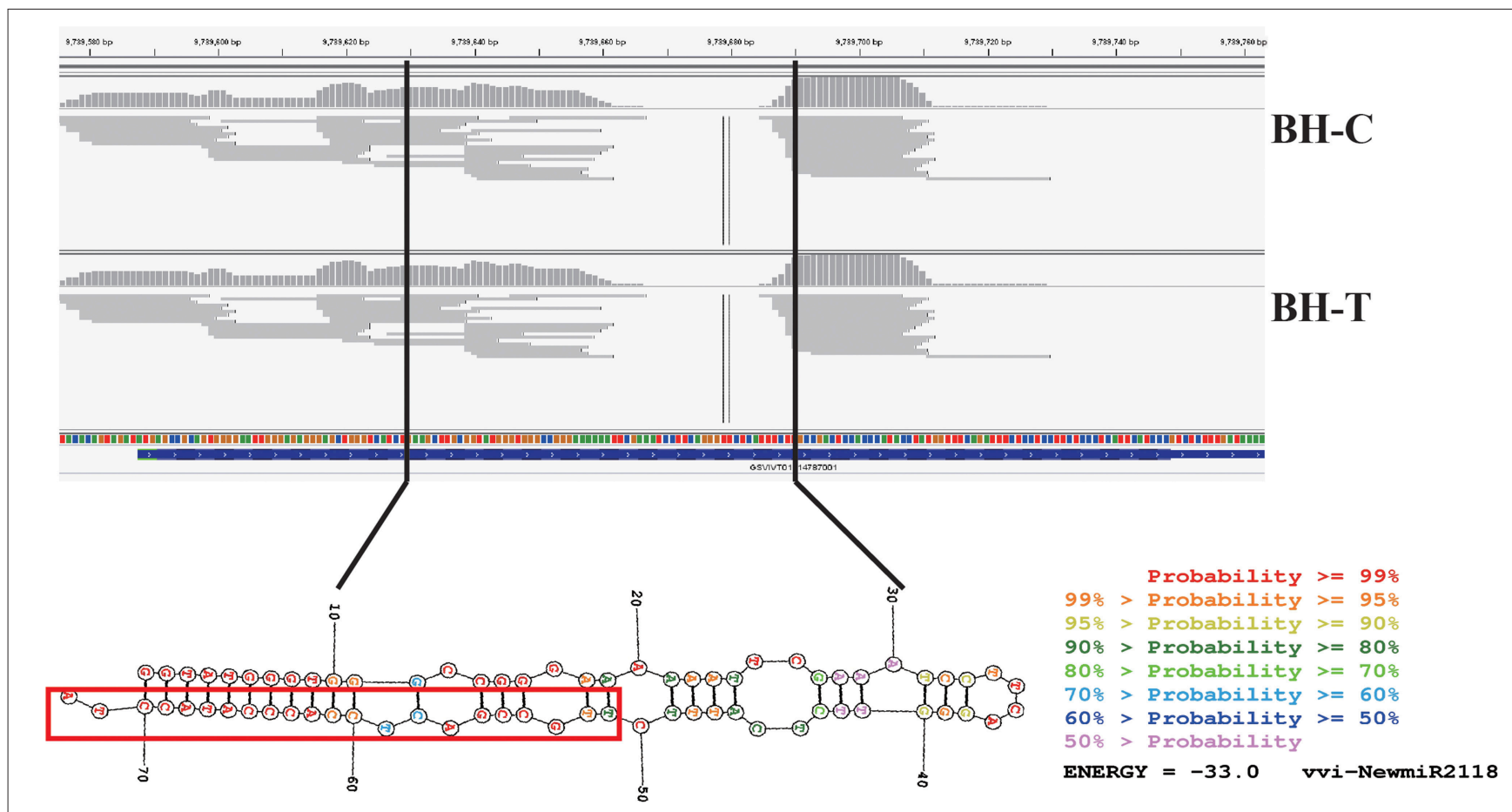

FIGURE 7 | Signature distribution of vvi-NewmiRNA2118 reads on Chinese wild grape genome and hairpin-like secondary structure of its precursor sequence. The color indicates base pair probabilities. The part in the box shows the mature sequence of vvi-NewmiRNA2118.

million years (Floyd and Bowman, 2004) and their targets primarily regulate the growth and development of plants, indicating the key role of miRNA in plant survival. However, conserved miRNAs do not equal that their functions are also conserved. For example, each of the three conserved miRNA families, miR473a, miR478a, and miR482 from Populus and rice has different functions ( $\mathrm{Lu}$ et al., 2005). In this study, we predicted targets which are not transcription factors for some conserved vvi-miRANs as well. For example, vvi-miR172c had a disease resistance target RPM-like (GSVIVT01028632001); vvi-miR156 and vvi-miR396 targeted disease resistance related protein GSVIVT01016962001 and TMV resistance protein nlike (GSVIVT01037219001). We suggest these conserved miRNA may be involved in stress responses. In contrast with conserved miRNAs, the targets of non-conserved miRNAs varied greatly, explaining their diverse roles in biological processes.

By semi-quantitative RT-PCR and qRT-PCR, we uncovered the expression patterns of a part of those identified miRNAs from Chinese wild $V$. pseudoreticulata after E. necator inoculation. Results showed that many miRNAs (vvi-miR159c, vvi-miR164, vvi-miR166f/g, vvi-miR396b, vvi-miR171, vvi-miR172, vvimiR156, vvi-miR166a, vvi-miR390) believed previously mainly involved in growth and development could be affected when suffered from fungi stress. Sunkar et al. (2012) also show that stress can significantly alter the expression profiles of most miRNAs function in growth and development. Our prediction of $\mathrm{R}$ or R-related target genes for vvi-miR156, 159, 171, 172, 390, and 396 may partially help explain their fluctuating expression. On the other hand, this may also provide information about why the normal growth status can be altered under biotic stress. For example, miR160, miR167 with targets of ARFs and miR393 with target of TIR1 are all up-regulated, while miR390 is down regulated under a range of stress conditions. Nevertheless, their common effect is the suppression of auxin signaling and growth (Sunkar et al., 2012).

In terms of the expression pattern of vvi-miRNAs, our results were not consistent when analyzed by miRDeep-P, semiquantitive RT-PCR, and qRT-PCR. The most likely reason comes from samples used for sRNA high-throughput sequencing. When we built the "Baihe-35-1" library under E. necator stress, we mixed equal total RNA extracted from all individual leave samples inoculated with $E$. necator for different time. It may be that differential expression of some vvi-miRNAs has been balanced by opposite-going changes at different time points.

The $\mathrm{R}$ genes are very important in plant genome. NBSLRR genes form overwhelming majority of them. NBS-LRR proteins are typically intracellular, soluble proteins with three domains: an N-terminal signaling domain, a central nucleotide binding site domain, and C-terminal leucine-rich repeats (LRR) (Jones and Dangl, 2006). Nucleotide binding site (NBS) contains three domains: p-loop (kinase-a), kinase-2a, and kinase3a. The signature sequence of the p-loop domain is GM (G/P)GXGKTT(a/r). According to Velasco et al. (2007), several clusters of NBS genes mapped to chromosomal regions were previously assigned to genetic resistance to fungal diseases such as downy and powdery mildews in grape. Many targets of miR2118 are often NBS-LRR genes, implying that miR2118 
A

miR2118a,c (Nelumbo nucifera)
gma-miR2118a
pvu-miR2118
mtr-miR2118a
miR2118e (Liriodendron chinense)
mdm-miR2118a
osa-miR2118a
zma-miR2118a
bdi-miR2118a
ata-miR2118a-5p
vvi-NewmiR2118
hbr-miR2118
sbi-miR2118-5p

- TTCCCAAGGCCTCCCATGCCG

- TTGCCGATTCCACCCATTCCT

- TTGCCGATTCCACCCATTCCT

- TTACCGATTCCACCCATTCCT

TTTACCTATTCCACCCATTCC.

- CTACCGATGCCACTAAGTCCC

- TTCTCGATGCCTCCCATTCCT

- TTCCTGATGCCTCTCATTCCT

- TTTCCGATGCCTCCCATTCCT

. TTCCTAATGTCTCCCATTCCT

- TTGCCGACTCCACCCATACCT

. GAAATGGGTGGATGGGAGTGA

. GGCATGGGAACATGTAGGAAG

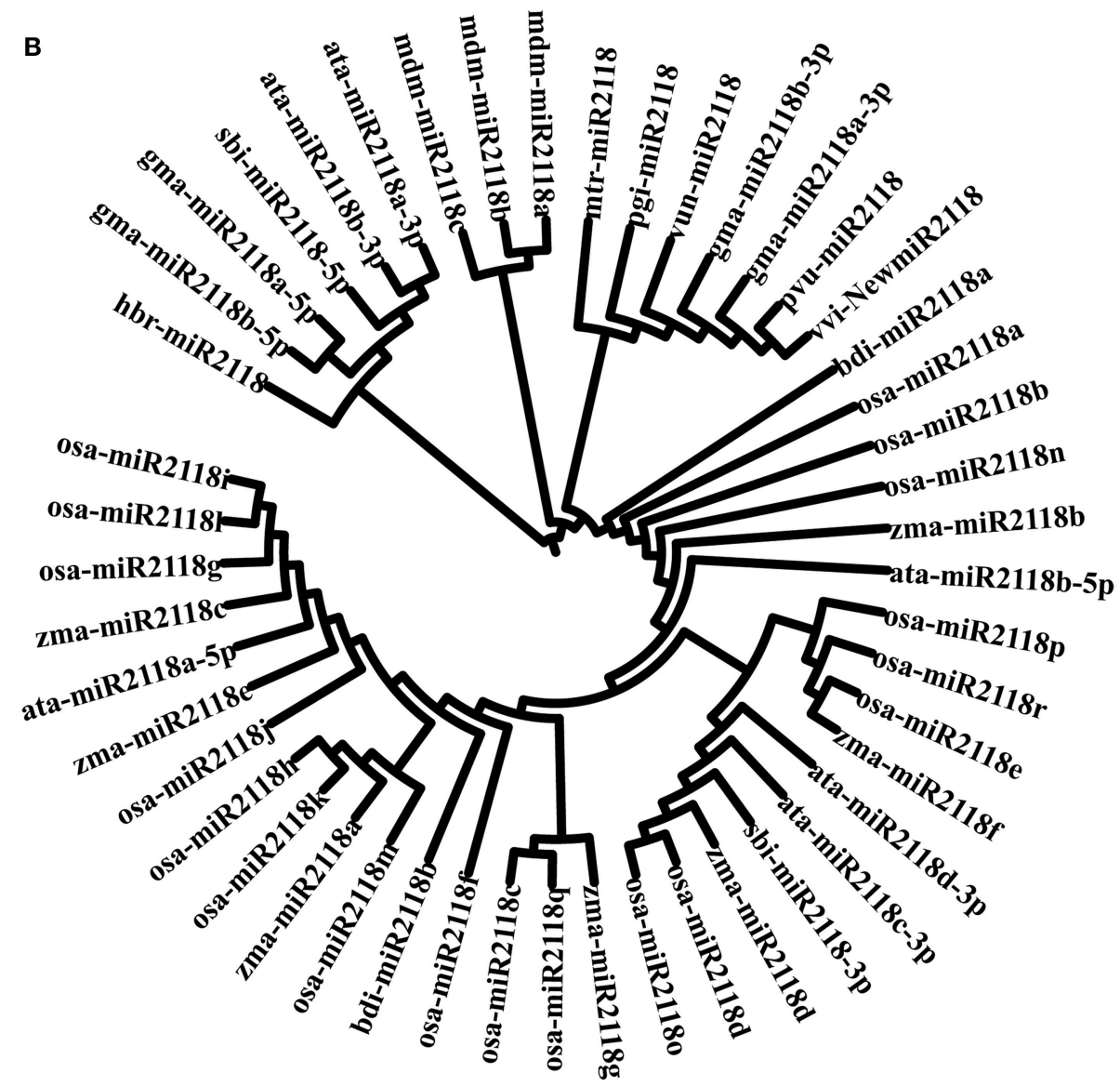

FIGURE 8 | Sequence alignment (A) and phylogeny analysis (B) for vvi-NewmiR2118. (A) 13 sequences for alignment were from the following species: Nelumbo nucifera (Zheng et al., 2013), Phaseolus vulgaris (Arenas-Huertero et al., 2009), Glycine max (Kulcheski et al., 2011), Medicago truncatula (Jagadeeswaran et al., 2009b), Zea mays (Johnson et al., 2009), Oryza sativa L. (Johnson et al., 2009), Brachypodium distachyon (Jeong et al., 2013), Aegilops tauschii (Jia et al., 2013), Malus domestica (Xia et al., 2012), Hevea brasiliensis L. (Lertpanyasampatha et al., 2012), sugarcane (Thiebaut et al., 2012), Liriodendron chinense (Wang et al., 2012), and Chinese wild Vitis pseudoreticulata. Mature miRNAs were shown from $5^{\prime}$ to $3^{\prime}$ end. Light gray and dark gray shading indicates the nucleotides were conserved in more than $70 \%$ or more than $50 \%(<70 \%)$ of the sequences, respectively. (B) There were 47 miRNA2118 sequences from miRBase 21.0 used for phylogeny analysis. As shown in the figure, annual and perennial plants clustered separately. vvi-NewmiR2118 was most closely related with that of Malus domestica and Panax ginseng, and have close relations with Phaseolus vulgaris.

plays a role in host-pathogen interactions (Jagadeeswaran et al., 2009b; Zhai et al., 2011; Shivaprasad et al., 2012). Research on tobacco and Glycine max shows that miR2118 usually targets the conserved regions, mainly p-loop sequences in these genes and functions by the generation of trans-acting siRNAs (Zhai et al., 2011).

To date, the function of miR2118 in grape and other plants is still poorly understood and its target mRNAs have 


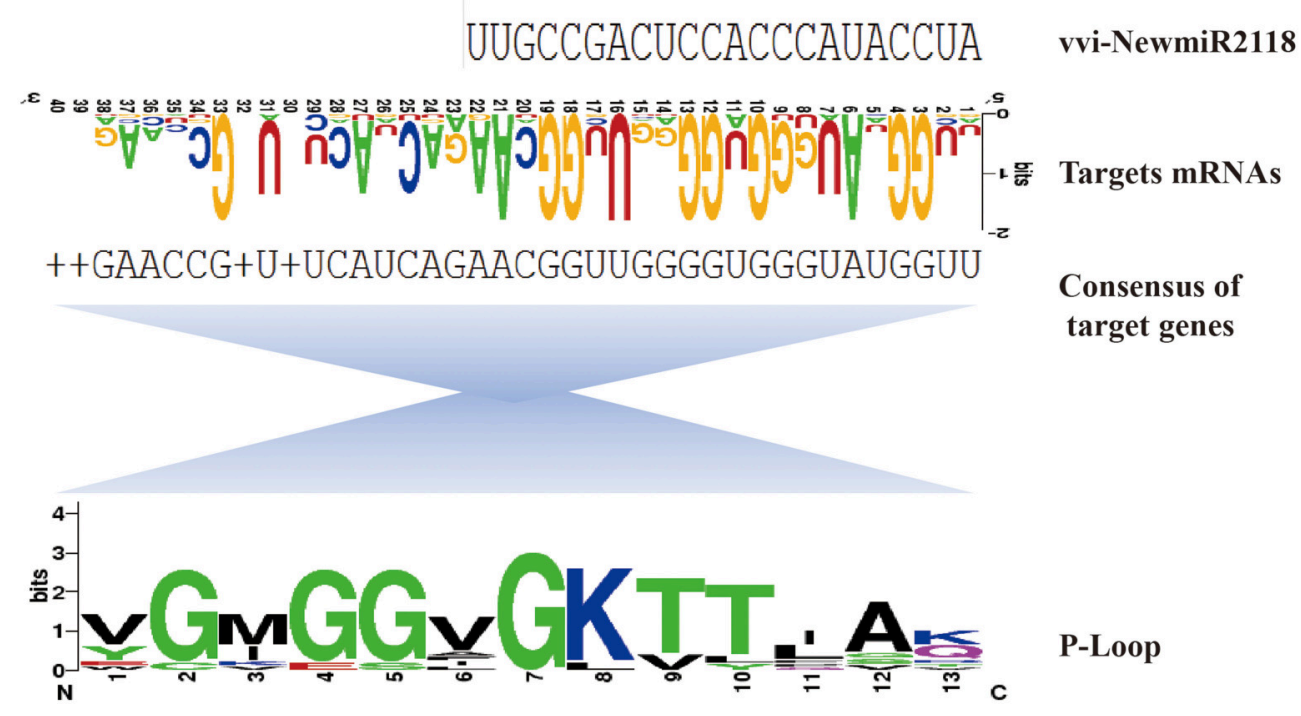

FIGURE 9 | Analysis of the interaction region of vvi-NewmiR2118 with NB-LRR targets. vvi-NewmiR2118 (first line, shown in $5^{\prime}$ to $3^{\prime}$ orientation) bound on the conserved p-loop motif (third line, translates of target genes) of NB-LRR targets (second line).
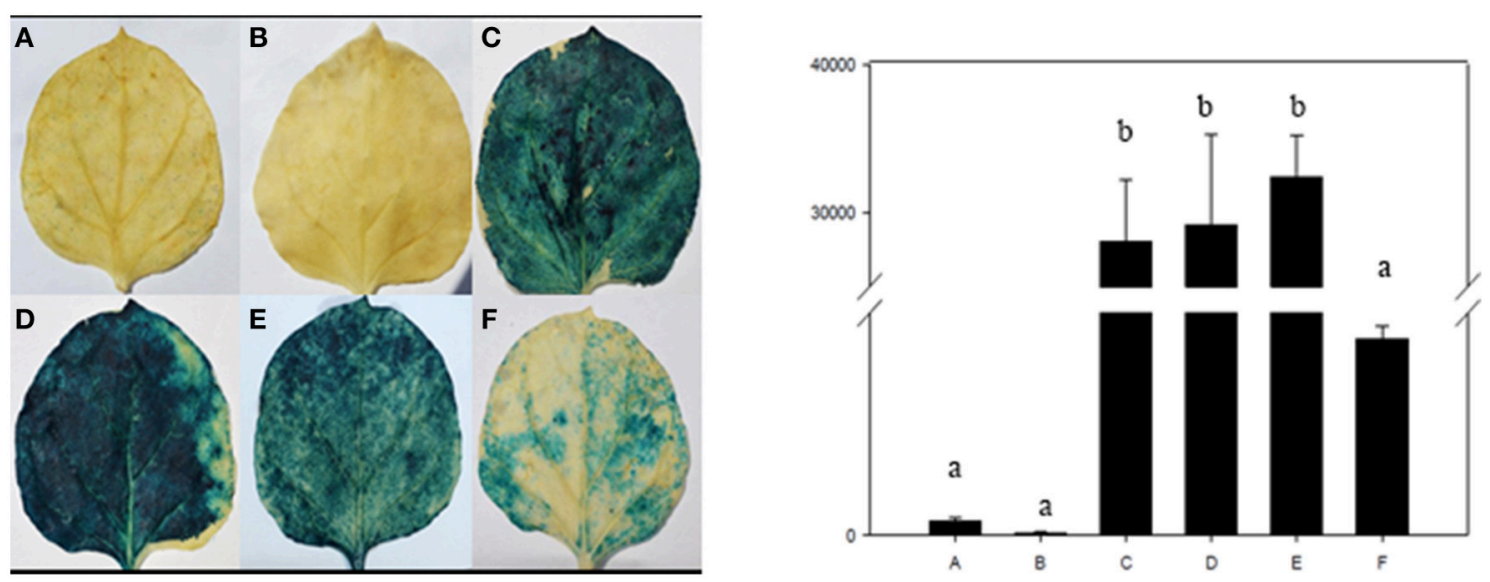

FIGURE 10 | GUS accumulation by histochemical staining (left) and fluorescence quantitative assay (right) of transiently transformed tobacco leaves mediated by Agrobacterium : (A) penetration buffer, (B) Pro35S:MIR2118, (C) Pro35S:GUS, (D) Pro35S: 2 RPP13-GUS, (E) Pro35S:MIR2118 and Pro35S:GUS $(\mathrm{V} / \mathrm{V}=5: 1)$, and (F) Pro35S:MIR2118 and Pro35S: $\triangle$ RPP13-GUS $(\mathrm{V} / \mathrm{V}=5: 1)$.

not been identified. In our research, we first identified 22nt long vvi-NewmiR2118 in wild Chinese V. pseudoreticulata and its expression was very high. When the wild grape suffers fungi attack, vvi-NewmiR2118 will be quickly downregulated. Moreover, we predicted targets for vvi-NewmiR2118. In accordance with many previous reports, these target genes were all $\mathrm{R}$ genes of the NBS-LRR type. Experiments also proved interaction of vvi-NewmiR2118 and resistance gene RPP13. However, it's still unknown how this miRNA functions in this pathway and if it will mediate the biosynthesis siRNAs. We analyzed the binding region of vvi-NewmiR2118 with several of its NBS-LRR targets and found that in grape, miR2118 also bound onto the p-loop domain of its targets. Hence we speculate that vvi-NewmiR2118 may regulate its target gene by producing pha-siRNA, however, more work is required to verify this idea.

\section{CONCLUSION}

To explore the defense mechanisms of grape to powdery mildew at the level of post-transcription regulation, we identified miRNAs from highly resistant wild grapevine "Baihe-35-1." Combined with sRNA high-throughput sequencing, bioinformatics and molecular biology technologies, we profiled and identified vvi-miRNAs that might be involved in powdery mildew resistance in Chinese wild V. pseudoreticulata. This not only revealed a novel layer of defense mechanisms 
against biotic stresses employed by grape, but also provided a new method for breeding disease resistant grapevines through transgenic technology.

\section{AUTHOR CONTRIBUTIONS}

YX conceived and initiated the work; LH designed the experiments, LH, HM carried out the experiments. LH analyzed the data and wrote the paper; KW, GX, and GL carried out and supervised the computational work. $\mathrm{LH}, \mathrm{KW}$ are co-authors.

\section{ACKNOWLEDGMENTS}

This research was supported by the National Natural Science Foundation of China (Grant No. 31272125), and the "948" Program, Ministry of Agriculture, China (grant No. 2011-G21).

\section{REFERENCES}

Akkurt, M., Welter, L., Maul, E., Töpfer, R., and Zyprian, E. (2007). Development of SCAR markers linked to powdery mildew (Uncinula necator) resistance in grapevine (Vitis vinifera L. and Vitis sp.). Mol. Breed. 19, 103-111. doi: 10.1007/s11032-006-9047-9

Alabi, O. J., Zheng, Y., Jagadeeswaran, G., Sunkar, R., and Naidu, R. A. (2012). High-throughput sequence analysis of small rnas in grapevine (vitis vinifera L.) affected by grapevine leafroll disease. Mol. Plant Pathol., 13, 1060-1076. doi: 10.1111/j.1364-3703.2012.00815.x

Arenas-Huertero, C., Pérez, B., Rabanal, F., Blanco-Melo, D., Rosa, C. D. L., Estrada-Navarrete, G., et al. (2009). Conserved and novel miRNAs in the legume phaseolus vulgaris in response to stress. Plant Mol. Biol. 70, 385-401. doi: 10.1007/s11103-009-9480-3

Baldrich, P., Cristina, P. P., Sonia, C., Wu, M. T., Hsing, Y., and Blanca, S. S. (2013). MicroRNAs (miRNAs) in Plant Innate Immunity: miR7695, a Novel miRNA from Rice Involved in Pathogen Resistance. Available online at: http://hdl.handle.net/10261/101851

Barakat, A., Wall, K., and Leebens-Mack, J. (2007). Large-scale identification of microRNAs from a basal eudicot (Eschscholzia californica) and conservation in flowering plants. Plant J. 51, 991-1003. doi: 10.1111/j.1365-313X.2007. 03197.x

Bradford, M. M. (1976). A rapid and sensitive method for the quantitation of microgram quantities of protein utilizing the principle of protein-dye binding. Anal. Biochem. 72, 248-254. doi: 10.1016/0003-2697(76)90527-3

Campo, S., Peris-Peris, C., Siré, C., Moreno, A. B., Donaire, L., Zytnicki, M., et al. (2013). Identification of a novel microRNA (miRNA) from rice that targets an alternatively spliced transcript of the Nramp6 (natural resistance-associated macrophage protein 6) gene involved in pathogen resistance. New Phytol. 199, 212-227. doi: 10.1111/nph.12292

Carra, A., Mica, E., Gambino, G., Pindo, M., Moser, C., Pè, M. E., et al. (2009). Cloning and characterization of small non-coding RNAs from grape. Plant J. 59, 750-763. doi: 10.1111/j.1365-313X.2009.03906.x

Chen, C., Ridzon, D. A., Broomer, A. J., Zhou, Z., Lee, D. H., Nguyen, J. T., et al. (2005). Real-time quantification of microRNAs by stem-loop rt-pcr. Nucleic Acids Res. 33, 179. doi: 10.1093/nar/gni178

Chen, X. (2005). MicroRNA biogenesis and function in plants. FEBS Lett. 579, 5923-5931. doi: 10.1016/j.febslet.2005.07.071

Dai, X., and Zhao, P. X. (2011). psRNAtarget: a plant small rna target analysis server. Nucleic Acids Res. 39, W155-W159. doi: 10.1093/nar/gkr319

Fahlgren, N., Howell, M. D., Kasschau, K. D., Chapman, E. J., Sullivan, C. M., Cumbie, J. S., et al. (2007). High-throughput sequencing of Arabidopsis microRNAs: evidence for frequent birth and death of MIRNA genes. PLoS ONE 2:e219. doi: 10.1371/journal.pone.0000219
Work was also supported by the Program for Innovative Research Team of Grape Germplasm Resource and Breeding (2013KCT25) and the Program for Fruit Breeding from the Cyrus Tang Foundation in Northwest A and F University to YX.

\section{SUPPLEMENTARY MATERIAL}

The Supplementary Material for this article can be found online at: http://journal.frontiersin.org/article/10.3389/fpls.2016. 00621

Table S1 | Primers used in this study.

Table S2 | vvi-miRNAs identified in the leaf of "Baihe-35-1."

Table S3 | vvi-miRNAs detected only in one library.

Table S4 | List of conserved miRNAs obtained from leaves of "Baihe-35-1."

Table S5 | Differentially expressed miRNAs from leaves of "Baihe-35-1" after inoculation with Erysiphe necator.
Floyd, S. K., and Bowman, J. L. (2004). Gene regulation: ancient microRNA target sequences in plants. Nature 428, 485-486. doi: 10.1038/428485a

He, P. C. (1999). Viticulture. Beijing: China Agriculture Press.

Jagadeeswaran, G., Saini, A., Sunkar, R., and Hoyt, P. (2009a). Biotic and abiotic stress down-regulate miR398 expression in Arabidopsis. Planta 229, 1009-1014. doi: 10.1007/s00425-009-0889-3

Jagadeeswaran, G., Zheng, Y., Li, Y. F., Shukla, L. I., Matts, J., et al. (2009b). Cloning and characterization of small RNAs from Medicago truncatula reveals four novel legume-specific microRNA families. New Phytol. 184, 85-98. doi: 10.1111/j.1469-8137.2009.02915.x

Jefferson, R. A., Kavanagh, T. A., and Bevan, M. W. (1987). Gus fusions: betaglucuronidase as a sensitive and versatile gene fusion marker in higher plants. Embo J. 6, 3901-3907.

Jeong, D. H., Schmidt, S. A., Rymarquis, L. A., Park, S., Ganssmann, M., German, M. A., et al. (2013). Parallel analysis of RNA ends enhances global investigation of microRNAs and target RNAs of brachypodium distachyon. Genome Biol. 14, R145-R145. doi: 10.1186/gb-2013-14-12-r145

Jia, J., Zhao, S., Kong, X., Li, Y., Zhao, G., He, W., et al. (2013). Aegilops tauschii draft genome sequence reveals a gene repertoire for wheat adaptation. Nature 496, 91-95. doi: 10.1038/nature12028

Johnson, C., Kasprzewska, A., Tennessen, K., Fernandes, J., Nan, G. L., Walbot, V., et al. (2009). Clusters and superclusters of phased small RNAs in the developing inflorescence of rice. Genome Res. 19, 1429-1440. doi: 10.1101/gr.089854.108

Jones, J. D. G., and Dangl, J. L. (2006). The plant immune system. Nature 444, 323-329. doi: 10.1038/nature05286

Jones-Rhoades, M. W., Bartel, D. P., and Bartel, B. (2006). MicroRNAs and their regulatory roles in plants. Annu. Rev. Plant Biol. 57, 19-53. doi: 10.1146/annurev.arplant.57.032905.105218

Kulcheski, F. R., Oliveira, L. F. V., Molina, L. G., Almerão, M. P., Rodrigues, F. A., Marcolino, J., et al. (2011). Identification of novel soybean microRNAs involved in abiotic and biotic stresses. BMC Genomics 12:307. doi: 10.1186/1471-216412-307

Lertpanyasampatha, M., Gao, L., Kongsawadworakul, P., Viboonjun, U., Chrestin, H., Liu, R., (2012). Genome-wide analysis of MicroRNAs in rubber tree (Hevea brasiliensis L.) using high-throughput sequencing. Planta 236, 437-445.

Lu, S., Sun, Y. H., Shi, R., Clark, C., Li, L., and Chiang, V. L. (2005). Novel and mechanical stress-responsive MicroRNAs in Populus trichocarpa that are absent from Arabidopsis. Plant Cell 17, 2186-2203. doi: 10.1105/tpc.105.033456

Mallory, A. C., and Vaucheret, H. (2006). Functions of microRNAs and related small RNAs in plants. Nat. Genet. 38, 850-850. doi: 10.1038/ng0706-850b

Mica, E., Piccolo, V., Delledonne, M., Ferrarini, A., Pezzotti, M., Casati, C., et al. (2009). High throughput approaches reveal splicing of primary microRNA transcripts and tissue specific expression of mature microRNAs in vitis vinifera. BMC Genomics 10:558. doi: 10.1186/1471-2164-10-558 
Morin, R. D., Aksay, G., Dolgosheina, E., Ebhardt, H. A., Magrini, V., Mardis, E. R., et al. (2008). Comparative analysis of the small rna transcriptomes of pinus contorta and oryza sativa. Genome Res. 18, 571-584. doi: 10.1101/gr.68 97308

Moxon, S., Jing, R., Szittya, G., Schwach, F., Rusholme Pilcher, R. L., Moulton, V., et al. (2008). Deep sequencing of tomato short rnas identifies microRNAs targeting genes involved in fruit ripening. Genome Res. 18, 1602-1609. doi: 10.1101/gr.080127.108

Navarro, L., Dunoyer, P., Jay, F., Arnold, B., Dharmasiri, N., Estelle, M., et al. (2006). A plant miRNA contributes to antibacterial resistance by repressing auxin signaling. Science 312, 436-439. doi: 10.1126/science.1126088

Navarro, L., Jay, F., Nomura, K., He, Y. S., and Voinnet, O. (2008). Suppression of the microRNA pathway by bacterial effector proteins. Science 321, 964-967. doi: $10.1126 /$ science.1159505

Niu, Q. W., Lin, S. S., Reyes, J. L., Chen, K. C., Wu, H. W., Yeh, S. D., et al. (2006). Expression of artificial microRNAs in transgenic Arabidopsis thaliana confers virus resistance. Nat. Biotechnol. 25, 1420-1428. doi: 10.1038/nbt1255

Pantaleo, V., Szittya, G., Moxon, S., Miozzi, L., Moulton, V., Dalmay, T., et al. (2010). Identification of grapevine microRNAs and their targets using highthroughput sequencing and degradome analysis. Plant J. 62, 960-976. doi: 10.1111/j.0960-7412.2010.04208.x

Qiu, D., Pan, X., Wilson, I. W., Li, F., Liu, M., Teng, W., et al. (2009). High throughput sequencing technology reveals that the taxoid elicitor methyl jasmonate regulates microRNA expression in chinese yew (taxus chinensis). Gene 436, 37-44. doi: 10.1016/j.gene.2009.01.006

Rajagopalan, R., Vaucheret, H., Trejo, J., and Bartel, D. P. (2006). A diverse and evolutionarily fluid set of microRNAs in Arabidopsis thaliana. Genes Dev. 20, 3407-3425. doi: 10.1101/gad.1476406

Reinhart, B. J., Weinstein, E. G., Rhoades, M. W., Bartel, B., and Bartel, D. P. (2002). MicroRNAs in plants. Genes Dev. 16, 1616-1626. doi: 10.1101/gad.1004402

Richter, H., Pezet, R., Viret, O., and Gindro, K. (2006). Characterization of 3 new partial stilbene synthase genes out of over 20 expressed in Vitis vinifera during the interaction with Plasmopara viticola. Physiol. Mol. Plant Pathol. 67, 248-260. doi: 10.1016/j.pmpp.2006.03.001

Ruiz-Ferrer, V., and Voinnet, O. (2009). Roles of plant small RNAs in biotic stress responses. Annu. Rev. Plant Biol. 60, 485-510. doi: 10.1146/annurev.arplant.043008.092111

Shivaprasad, P. V., Chen, H. M., Patel, K., Bond, D. M., Santos, B. A., and Baulcombe, D. C. (2012). A microRNA superfamily regulates nucleotide binding site-leucine-rich repeats and other mrnas. Plant Cell 24, 859-874. doi: 10.1105/tpc.111.095380

Song, C., Wang, C., Zhang, C., Korir, N. K., Yu, H., Ma, Z., et al. (2010). Deep sequencing discovery of novel and conserved microRNAs in trifoliate orange (Citrus trifoliata). BMC Genomics 11:431. doi: 10.1186/1471-2164-11-431

Sparkes, I. A., Runions, J., Kearns, A., and Hawes, C. (2006). Rapid, transient expression of fluorescent fusion proteins in tobacco plants and generation of stably transformed plants. Nat. Protoc. 1, 2019-2025. doi: 10.1038/nprot.2006.286

Sunkar, R., Kapoor, A., and Zhu, J. K. (2006). Posttranscriptional induction of two $\mathrm{Cu} / \mathrm{Zn}$ superoxide dismutase genes in Arabidopsis is mediated by downregulation of miR398 and important for oxidative stress tolerance. Plant Cell 18, 2051-2065. doi: 10.1105/tpc.106.041673

Sunkar, R., Li, Y. F., and Jagadeeswaran, G. (2012). Functions of microRNAs in plant stress responses. Trends Plant Sci. 17, 196-203. doi: 10.1016/j.tplants.2012.01.010

Thiebaut, F., Grativol, C., Carnavale-Bottino, M., Rojas, C. A., Tanurdzic, M., Farinelli, L., et al. (2012). Computational identification and analysis of novel sugarcane microRNAs. BMC Genomics 13:290. doi: 10.1186/1471-2164-13-290

Velasco, R., Zharkikh, A., Troggio, M., Cartwright, D. A., Cestaro, A., Pruss, D., et al. (2007). A high quality draft consensus sequence of the genome of a heterozygous grapevine variety. PLOS ONE 2:e1326. doi: 10.1371/journal.pone.0001326

Wan, Y. Z., Schwaniger, H., He, P. C., and Wang, Y. J. (2007). Comparison of resistance to powdery mildew and downy mildew in Chinese wild grapes.
Vitis 46, 132-136. Available online at: http://pub.jki.bund.de/index.php/VITIS/ article/view/4245/4197

Wang, C., Shangguan, L., Kibet, K. N., Wang, X., Han, J., Song, C., et al. (2011). Characterization of microRNAs identified in a table grapevine cultivar with validation of computationally predicted grapevine miRNAs by miR-RACE. PLoS ONE 6:e21259. doi: 10.1371/journal.pone.0021259

Wang, K., Li, M., Gao, F., Li, S. Q., Zhu, Y. G., and Yang, P. F. (2012). Identification of conserved and novel microRNAs from Liriodendron chinense floral tissues. PLoS ONE 7:e44696. doi: 10.1371/journal.pone.0044696

Wang, Y. J., Liu, Y. L., He, P. C., Lamikanra, O., and Lu, J. (1998). Resistance of Chinese Vitis species to Elsinoe ampelina (de Bary) Shear. HortScience 33, 123-126.

Wang, Z., Qu, S., Zhang, Z., and Zhang, J. (2004). A fast method for total RNA extraction from the tissue culture material of malus sp. J. Fruit Sci. 4, 026. doi: 10.13925/j.cnki.gsxb.2004.04.026

Weng, K., Li, Z. Q., Liu, R. Q., Wang, L., Wang,Y. J., and Xu,Y. (2014). Transcriptome of Erysiphe necator-infected Vitis pseudoreticulata leaves provides insight into grapevine resistance to powdery mildew. Horticulture Res 1, 14049. doi: 10.1038/hortres.2014.49

Xia, R., Zhu, H., An, Y. Q., Beers, E. P., and Liu, Z. R. (2012). Apple miRNAs and tasiRNAs with novel regulatory networks. Genome Biol. 13:R47. doi: 10.1186/gb-2012-13-6-r47

Xie, K., Wu, C., and Xiong, L. (2006). Genomic organization, differential expression, and interaction of squamosa promoter-binding-like transcription factors and microRNA156 in rice. Plant Physiol. 142, 280-293. doi: 10.1104/pp.106.084475

Xu, M. J., Liu, Q., Nisbet, A. J., Cai, X. Q., Chao, Y., and Lin, R. Q. (2010). Identification and characterization of microRNAs in clonorchis sinensis of human health significance. BMC Genomics 11:521. doi: 10.1186/14712164-11-521

Yan, K., Liu, P., Wu, C. A., Yang, G. D., Xu, R., Guo, Q. H., et al. (2012). Stress-induced alternative splicing provides a mechanism for the regulation of microRNA processing in Arabidopsis thaliana. Mol. Cell 48, 521-531. doi: 10.1016/j.molcel.2012.08.032

Yang, X., and Li, L. (2011). Mirdeep-p: a computational tool for analyzing the microRNA transcriptome in plants. Bioinformatics 27, 2614-2615. doi: 10.1093/bioinformatics/btr430

Yang, X., Zhang, H., and Li, L. (2012). Alternative mRNA processing increases the complexity of microRNA-based gene regulation in Arabidopsis. Plant J. 70, 421-431. doi: 10.1111/j.1365-313X.2011.04882.x

Zhai, J., Jeong, D. H., De Paoli, E., Park, S., Rosen, B. D., Li, Y., et al. (2011) MicroRNAs as master regulators of the plant NB-LRR defense gene family via the production of phased, trans-acting siRNAs. Genes Dev. 25, 2540-2553. doi: 10.1101/gad.177527.111

Zhang, J., Wang, Y., Wang, X., Yang, K., and Jinxiao, A. Y. (2003). An improved method for rapidly extracting total RNA from vitis. J. Fruit Sci. 3, 178-181. doi: 10.13925/j.cnki.gsxb.2003.03.006

Zheng, Y., Jagadeeswaran, G., Gowdu, K., Wang, N., Li, S., Ming, R., et al. (2013). Genome-wide analysis of microRNAs in sacred lotus, nelumbo nucifera (gaertn). Trop. Plant Biol. 6, 117-130. doi: 10.1007/s12042-013-9127-Z

Zhou, D., Xin, Z., Yi, L., Zhang, Z. H., and Zhen S (2010). A GO analysis toolkit for the agricultural community. Nucleic Acids Res. 38, W64-W70. doi: $10.1093 / \mathrm{nar} / \mathrm{gkq} 310$

Conflict of Interest Statement: The authors declare that the research was conducted in the absence of any commercial or financial relationships that could be construed as a potential conflict of interest.

Copyright (C) 2016 Han, Weng, Ma, Xiang, Li, Wang, Liu and Xu. This is an openaccess article distributed under the terms of the Creative Commons Attribution License (CC BY). The use, distribution or reproduction in other forums is permitted, provided the original author(s) or licensor are credited and that the original publication in this journal is cited, in accordance with accepted academic practice. No use, distribution or reproduction is permitted which does not comply with these terms. 